\title{
The Role of $\mathrm{SrTiO}_{3}$ Phonon Penetrating into thin FeSe Films in the Enhancement of Superconductivity
}

\author{
Shuyuan Zhang, ${ }^{1}$ Jiaqi Guan, ${ }^{1}$ Xun Jia, ${ }^{1}$ Bing Liu, ${ }^{1}$ Weihua Wang, ${ }^{1}$ Fangsen Li,${ }^{2}$ Lili Wang,, 2 , , Xucun \\ Ma, ${ }^{2,3}$ Qikun Xue, ${ }^{2,3}$ Jiandi Zhang, ${ }^{4}$ E. W. Plummer, ${ }^{4}$ Xuetao Zhu, ${ }^{1, \dagger}$ and Jiandong Guo ${ }^{1,3}$, \\ ${ }^{1}$ Beijing National Laboratory for Condensed Matter Physics and Institute of Physics, \\ Chinese Academy of Sciences, Beijing 100190, China \\ ${ }^{2}$ State Key Laboratory of Low-Dimensional Quantum Physics, \\ Department of Physics, Tsinghua University, Beijing 100084, China \\ ${ }^{3}$ Collaborative Innovation Center of Quantum Matter, Beijing 100871, China \\ ${ }^{4}$ Department of Physics and Astronomy, Louisiana State University, Baton Rouge, LA 70808, USA
}

(Dated: June 8, 2021)

\begin{abstract}
The significant role of interfacial coupling on the superconductivity enhancement in FeSe films on $\mathrm{SrTiO}_{3}$ has been widely recognized. But the explicit origination of this coupling is yet to be identified. Here by surface phonon measurements using high resolution electron energy loss spectroscopy, we found electric field generated by Fuchs-Kliewer (F-K) phonon modes of $\mathrm{SrTiO}_{3}$ can penetrate into FeSe films and strongly interact with electrons therein. The mode-specific electronphonon coupling (EPC) constant for the $\sim 92 \mathrm{meV} \mathrm{F-K}$ phonon is $\sim 0.25$ in the single-layer FeSe on $\mathrm{SrTiO}_{3}$. With increasing FeSe thickness, the penetrating field intensity decays exponentially, which matches well the observed exponential decay of the superconducting gap. It is unambiguously shown that the $\mathrm{SrTiO}_{3}$ F-K phonon penetrating into FeSe is essential in the interfacial superconductivity enhancement.
\end{abstract}

The recent discovery of high-temperature superconductivity in 1 unit cell (u.c.) FeSe films on $\mathrm{SrTiO}_{3}$ (STO) substrate [1, 2] has attracted a lot of attention, since the superconducting transition temperature $\left(\mathrm{T}_{C}\right)$ is significantly raised to $\sim 60-75 \mathrm{~K}[36$, even over $100 \mathrm{~K}[7$, from $8 \mathrm{~K}$ for the bulk FeSe 8 . Various experiments have been performed to uncover the veiled mechanism of the $\mathrm{T}_{C}$ enhancement in this system. Although no explicit conclusion has been reached, two factors are widely believed to be essential - electron doping to FeSe films and the coupling at interface between FeSe and STO.

The significance of electron doping in the interfacial superconductivity enhancement has been evidenced by the high resolution angle resolved photoemission spectroscopy (ARPES) measurements. Extensive annealing drives electrons transferred from STO substrate to the 1 u.c. FeSe thus increases the electron density in the film, and broadens the superconducting gap accordingly [4, 9, 10. In fact the superconducting 1 u.c. FeSe/STO has similar Fermi surface as other two typical electron doped iron-based superconductors, e.g., $\mathrm{A}_{x} \mathrm{Fe}_{2} \mathrm{Se}_{2}$ $(\mathrm{A}=\mathrm{K}, \mathrm{Cs})$ [11] and $(\mathrm{Li}, \mathrm{Fe}) \mathrm{OHFeSe}$ [12. The electron density in thick FeSe films can also be tuned by alkalimetal doping [13 16] or by ion-liquid gate tuning [17-19]. Even though the electron density can be raised up to the value as high as that in the $1 \mathrm{u}$. c. FeSe/STO with $\mathrm{T}_{C}$ of $60-75 \mathrm{~K}$, the superconductivity in thick FeSe film is always weaker (with the highest reported $\mathrm{T}_{C}$ of $\sim 48$ $\mathrm{K}$ [18]) than that in the 1 u.c. FeSe/STO. It is indicated that, besides electron doping, there must be other factor(s) that is responsible for the superconductivity enhancement in FeSe/STO.

The importance of the interfacial coupling has been directly evidenced by the substrate selection behavior of the superconductivity enhancement. On oxide substrates such as $\mathrm{SrTiO}_{3}$ or $\mathrm{BiTiO}_{3}, \mathrm{~T}_{C}$ is strongly enhanced no matter what crystal orientation, crystal symmetry or lattice constant the substrates have 20 23; while when thin FeSe films are grown on graphene/SiC(0001) [16, 24, 25], even though similar electron density is accomplished by $\mathrm{K}$-doping, the maximum superconducting gap is always smaller than that of 1 u.c. FeSe/STO. Moreover, a ferroelectric transition of STO was observed by Raman spectroscopy at $\sim 50 \mathrm{~K}$, quite close to the superducting $\mathrm{T}_{C}$ of the 1 u.c. FeSe/STO, implying the possible correlation between the substrate lattice and the superconductivity enhancement at the interface [26]. Recent high resolution ARPES measurements show that each energy band of the 1 u.c. FeSe is replicated at $\sim 100 \mathrm{meV}$ higher binding energy, which can be attributed to the interaction with the STO optical phonon around $80 \mathrm{meV}[5]$. Theoretical analyses further suggested that this strong interaction between the high energy STO phonon and the electrons in FeSe is responsible for the $\mathrm{T}_{C}$ enhancement [5].

All these findings seem to correlate the interfacial coupling with some unique properties of the oxide substrates, especially the optical phonon mode. By measuring the properties directly related to the electronic state, such as the electronic structure or the electron's life time, the electron-phonon coupling (EPC) constant can be roughly estimated [5, 27]. But the involved phonon mode cannot be identified directly, which limits our understanding of the underlying physics. In this letter, we report on surface phonon measurements for thin FeSe films grown on STO, obtained by high resolution electron energy loss spectroscopy (HREELS). From the phonon perspective, 
TABLE I. The assignments of the energy loss features

\begin{tabular}{c|c|c}
\hline \hline Feature & Energy $(\hbar \omega)$ & Assignment \\
\hline$\eta$ & $\sim 0-7 \mathrm{meV}$ & acoustic phonon of FeSe \\
$\sigma$ & $\sim 20 \mathrm{meV}$ & optical phonon of FeSe \\
$\zeta$ & $\sim 32 \mathrm{meV}$ & optical phonon of FeSe \\
$\beta$ & $\sim 60 \mathrm{meV}$ & F-K surface phonon of STO \\
$\alpha$ & $\sim 92 \mathrm{meV}$ & F-K surface phonon of STO \\
\hline \hline
\end{tabular}

our results clearly reveal that electric field generated by the Fuchs-Kliewer (F-K) 28] surface phonon modes of STO can penetrate into thin FeSe films and strongly interact with the electrons in the FeSe layer with a modespecific coupling constant value $\lambda_{\alpha} \sim 0.25$ for the $\sim 92$ meV branch. The incomplete screening of the electric field associated with the F-K modes is the key to induce the interfacial coupling between FeSe and STO, and further result in the superconductivity enhancement.

Thin FeSe films with different thickness (1 u.c., 2 u.c., 3 u.c. and 10 u.c.) were grown on STO (001) surface by molecular beam epitaxy following the procedures reported in Ref. 1, 2. For convenience, these samples will be referred to as 1uc-FeSe/STO, 2uc-FeSe/STO, etc., respectively. All samples were characterized by scanning tunneling microscopy to confirm the high growth quality. And the superconducting property of 1uc-FeSe/STO was verified by scanning tunneling spectroscopy and ARPES. The determined superconducting gap $(\Delta)$ is around 20 $\mathrm{meV}$ and $\mathrm{T}_{C}$ is in the range of $60-70 \mathrm{~K}$. The details about the sample preparation and characterization are described in the Supplementary Material 29].

As a surface sensitive technique, HREELS is an ideal tool to explore the substrate effects on epitaxial thin films. HREELS measurements, carried out by the recently developed 2D-HREELS system [31, were performed on all samples in the temperature range from 35 $\mathrm{K}$ to $300 \mathrm{~K}$. Fig. 1 shows the HREELS results of 1uc$\mathrm{FeSe} / \mathrm{STO}$ at room temperature. Five different energy loss features are observed. The assignments [29] of these features are shown in Table. [1]

As exhibited in the energy distribution curves (EDCs) of Fig. 1 (b), the most prominent features are the $\alpha$ and $\beta$ modes corresponding to the F-K surface phonons of STO 32. Normally the phonons of substrate covered by a metal film should not have been detected by HREELS. But these F-K phonon modes are always accompanied with large dipole oscillations with electric field extending out of the STO surface [28, as if the F-K modes could penetrate into the epitaxial metal film. In HREELS measurement, the incident electrons are so sensitive to the dipole field 33 . that the detection of the penetrating electric field from the substrate can be realized. The penetration makes it possible that the electrons in FeSe films interact with the STO phonons. Moreover, as demon-

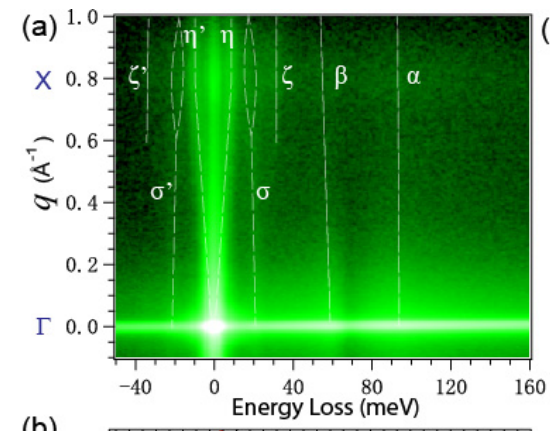

(b)
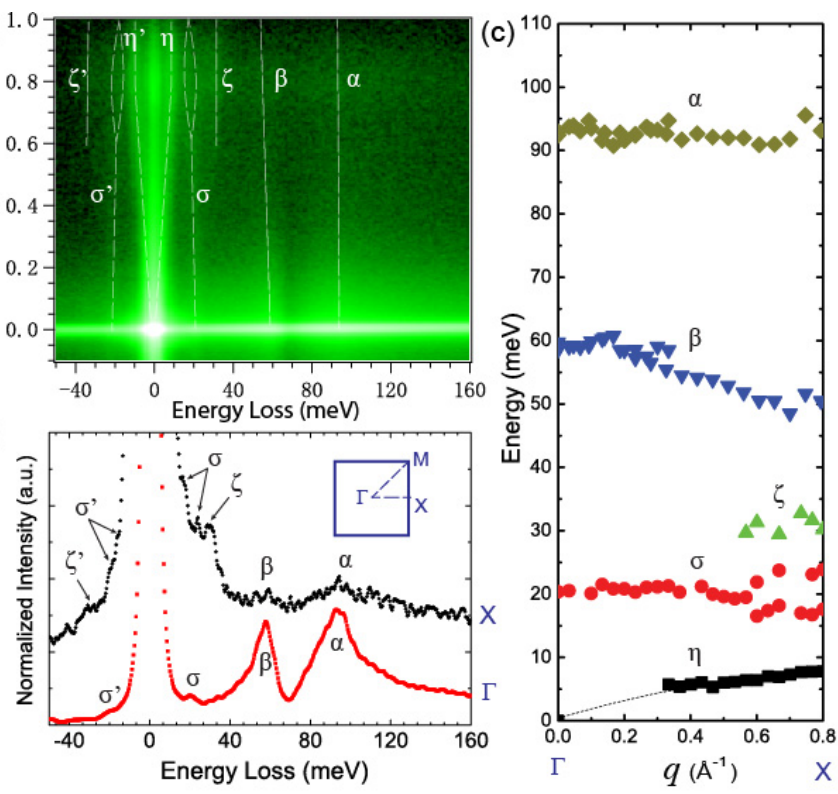

(d)
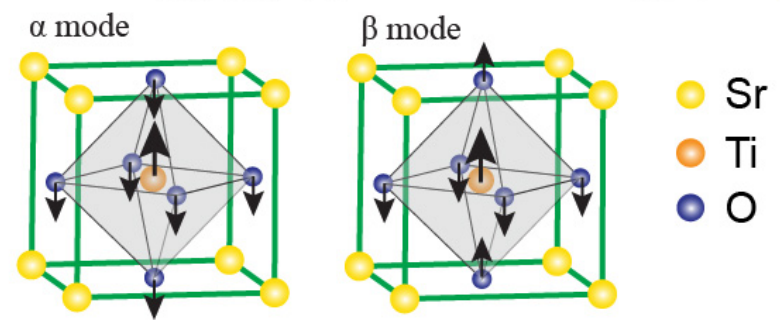

FIG. 1. HREELS results of the 1uc-FeSe/STO sample at room temperature. (a) 2D energy-momentum mapping along the $\Gamma$-X direction. Five different phonon modes with positive energy loss are labeled by $\alpha, \beta, \sigma, \zeta$, and $\eta$, respectively. Dashed lines are provided to guide the eye. The corresponding negative energy loss features correspond to their anti-Stokes peaks, which are labeled by $\sigma^{\prime}, \eta^{\prime}$, etc. (b) EDCs at $\Gamma$ point and $\mathrm{X}$ point with the inset shows the Brillouin zone. (c) Phonon dispersions from the results of panel(a). (d) Ionic vibrations of the $\alpha$ and $\beta$ modes 30 .

strated in Fig. 1 (c), the energy of the $\alpha$ mode is dispersionless, i.e. almost a constant of $\sim 92 \mathrm{meV}$ within the experimental resolution of about $3 \mathrm{meV}$ at different momentum values, which corroborates the ARPES observation [5] that the replica band is separated from the main band by $\sim 100 \mathrm{meV}$ all through the Brillouin Zone. Our analysis reveals that this $\alpha$ mode induces rather strong $\mathrm{EPC}$, and thus in the following we will focus on the $\alpha$ phonon branch as a representative. The contribution of the $\beta$ mode, with relatively weak EPC, is described in the Supplementary Material [29].

The HREELS measurements on 1uc FeSe/STO and bare STO are carried out at various temperatures and the temperature dependence of the energy and line width of the $\alpha$ phonon branch are shown in Fig. 2. The phonon energy of bare STO exhibits a very small increase with increasing temperature. In particular, when we take into 


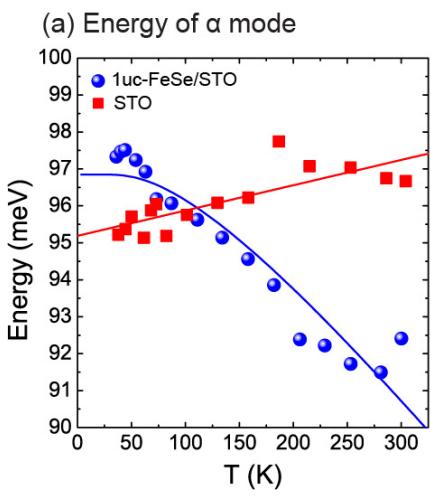

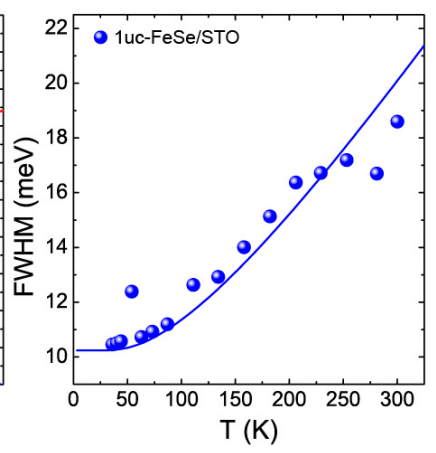

(b) Linewidth of a mode

FIG. 2. The temperature dependence of the (a) energy and (b) Full width at half maximum (FWHM) of the $\alpha$ phonon branch (blue: 1uc-FeSe/STO; red: bare STO). The dots are experimental data obtained by fitting the EDCs from the HREELS spectra at the $\Gamma$ point with Gaussian peaks. The red solid line is a linear fitting for bare STO, and the blue solid lines for 1uc-FeSe/STO represent the results of the phononphonon decay fitting described in the text.

account an instrument resolution of about $3 \mathrm{meV}$, it is negligible. This is consistent with the results in Refs. 34. and [35. The line width of bare STO is not shown here because it is overlapped with some other collective modes at low temperature and can not give credible results without critical analysis. This will be investigated in detail elsewhere. In contrast, the phonon energy as well as the line width of 1uc-FeSe/STO show a very strong temperature dependence. Obviously the growth of FeSe on STO changes the energy and its temperature-dependent behavior of the F-K phonon mode drastically. Since F-K phonon modes are strongly related to the dielectric response of STO, the observed T-dependence of the F-K mode reflects the coupling between the $\mathrm{F}-\mathrm{K}$ phonons and the electrons at the interface.

The energy for a specific phonon branch $\nu$ at temperature $T$ and momentum $\mathbf{q}$ can be written as a complex form 36 .

$$
\widetilde{\omega}(\mathbf{q}, \nu, T)=\operatorname{Re}[\widetilde{\omega}(\mathbf{q}, \nu, T)]-i \operatorname{Im}[\widetilde{\omega}(\mathbf{q}, \nu, T)],
$$

with the energy as the real part

$\operatorname{Re}[\widetilde{\omega}(\mathbf{q}, \nu, T)]=\omega_{0}(\mathbf{q}, \nu)+\Delta \omega_{V}(\mathbf{q}, \nu ; T)+\Delta \omega_{p p}(\mathbf{q}, \nu ; T)$,

and the line width (half width at half maximum) as the imaginary part

$$
\operatorname{Im}[\widetilde{\omega}(\mathbf{q}, \nu, T)]=\gamma_{e p}(\mathbf{q}, \nu)+\gamma_{p p}(\mathbf{q}, \nu ; \mathbf{T}),
$$

where $\omega_{0}(\mathbf{q}, \nu)$ is the harmonic phonon energy at $T=$ $0 \mathrm{~K}$ including the $T$-independent EPC contribution, $\Delta \omega_{V}(\mathbf{q}, \nu ; \mathbf{T})$ is the energy shift due to the change in the volume, and $\Delta \omega_{p p}(\mathbf{q}, \nu ; \mathbf{T})$ is the energy shift due to the anharmonic phonon-phonon interactions. The imaginary term $-i \operatorname{Im}[\widetilde{\omega}(\mathbf{q}, \nu, T)]$ describes the damping of the phonons, with contributions from both EPC $\gamma_{e p}(\mathbf{q}, \nu)$ and anharmonicity $\gamma_{p p}(\mathbf{q}, \nu ; \mathbf{T})$. Considering the anharmonic interaction usually lower the phonon energy at an elevated temperature, the slight temperature-dependence of the $\alpha$ phonon branch of bare STO should be mainly due to the volume change. However, when covered by FeSe, the significant energy decrease with increasing temperature of the $\alpha$ phonon branch should be attributed to strong anharmonic phonon-phonon interaction.

This anharmonic phonon-phonon interaction can be easily perceived in Table. [1 from the point of view of the energy conservation. One can immediately tell that the $\alpha$ mode decays into $\zeta$ and $\beta$ modes should be the most favorable decay channel. This conjecture is verified by simulating the anharmonic interaction via three different phonon decay models 29]. Since the $\alpha$ mode is dispersionless (shown in Fig,1), we set the energy to be qindependent in our models. It turns out the best fitting results are indeed from a model that the $\alpha$ mode with energy $\hbar \omega_{0}=92 \mathrm{meV}$ decays into another two optical phonon modes $\left(\zeta\right.$ and $\beta$ ) with lower energies $\hbar \omega_{1}=60$ $\mathrm{meV}$ and $\hbar \omega_{2}=32 \mathrm{meV}$, where the restriction of energy conservation $\hbar \omega_{0}=\hbar \omega_{1}+\hbar \omega_{2}$ is satisfied. In this model the temperature-dependent phonon energy $\hbar \omega(T)$ and line width $\gamma(T)$ are expressed as 37:

$$
\hbar \omega(T)=\hbar \omega_{a}+\hbar \omega_{b}\left(1+\frac{1}{e^{\hbar \omega_{1} / k_{B} T}-1}+\frac{1}{e^{\hbar \omega_{2} / k_{B} T}-1}\right),
$$

and

$$
\gamma(T)=\gamma_{e p}\left(1+\frac{1}{e^{\hbar \omega_{1} / k_{B} T}-1}+\frac{1}{e^{\hbar \omega_{2} / k_{B} T}-1}\right),
$$

where $\hbar \omega_{a}, \hbar \omega_{b}$, and $\gamma_{e p}$ are fitting parameters. The fitting results are plotted as blue solid lines in Fig. 2. This model explicitly clarifies the big difference of the anharmonic feature between STO and 1uc-FeSe/STO. The $32 \mathrm{meV} \zeta$ mode of FeSe, collaborating with the $60 \mathrm{meV}$ $\beta$ mode of STO, coincidentally renders an appropriate phonon decay channel for the $92 \mathrm{meV} \alpha$ mode. However, if there is no FeSe film, this decay channel is absent in STO substrate due to the restriction of energy conservation. Hence this anharmonic interaction characterizes part of the interfacial coupling. Its role in the superconductivity enhancement is not clear yet. Further studies are needed to figure out the explicit role of the anharmonicity.

The other part of the interfacial coupling, the penetrating STO F-K phonons interacting with the electrons in FeSe, is directly relevant to the superconductivity enhancement. The strength of this interaction can be characterized by a mode-specific EPC constant $\lambda(\mathbf{q}, \nu)$ for a single phonon mode of wave vector $\mathbf{q}$ and branch $\nu$, which is related to the EPC-induced line width $\gamma_{e p}(\mathbf{q}, \nu)$ by Allen's formula [38, 39.

$$
\lambda(\mathbf{q}, \nu)=\frac{2 \gamma_{e p}(\mathbf{q}, \nu)}{\pi N\left(E_{F}\right) \hbar^{2} \omega^{2}(\mathbf{q}, \nu)},
$$


where $\omega(\mathbf{q}, \nu)$ is the phonon frequency and $N\left(E_{F}\right)$ is the density of states for both spin in each unit cell at the Fermi energy.

To obtain the EPC constant from Allen's formula, the EPC-induced line width $\gamma_{e p}(\mathbf{q}, \nu)$ should be a prerequisite quantity. However, it is challenging to directly obtain the pure EPC induced phonon line widths from experiment, because the measured phonon line widths also contain additional contributions from anharmornic phononphonon interactions as shown in Eq.3. Only when the temperature-dependent anharmonic phonon-phonon interaction is correctly deducted, can the EPC-induced line width $\gamma_{e p}$ be determined and $\lambda$ be calculated. This can be accomplished from measurements of the temperature dependence of the phonon dispersions [36] (see Fig. 22). We extract $\gamma_{e p}$ for $\alpha$ mode through above anharmonic phonon decay model, as shown in Eq. 4 and Eq. 5, which gives $\gamma_{e p}(\alpha)=\gamma(T=0) \cong 5.1 \mathrm{meV}$, since there is no phonon-phonon interaction at $T=0$ and $\gamma(T=0)$ is the line width with EPC only. Independently, by analyzing the temperature-dependence of the phonon energy, we have also tried another approach using Kramers-Kronig relation to obtain $\gamma_{e p}(\alpha)$ 29. In this method, $\gamma_{p p}(\nu ; T)$ in Eq. 3 can be estimated through the Kramers-Kronig transformation of $\Delta \omega_{p p}(\nu ; T)$ in Eq. 2, which can be determined from a fitting of experimental data. Hence by subtracting the anharmonic contribution $\gamma_{p p}(\alpha ; T)$ from measured line width $\gamma(\alpha ; T)$, we obtained $\gamma_{e p}(\alpha) \cong 4.5$ meV [29].

On the other hand, a simple tight-binding model is applied to fit the electron band structure near the Fermi Surface to calculate the density of states $N\left(E_{F}\right)=$ $1.4 \times 10^{-3}(\mathrm{meV})^{-1} 29$. Thus the EPC constant of the $\alpha$ mode could be obtained from Eq $6, \lambda_{\alpha} \sim 0.25$. This coupling constant is significant for such a high energy phonon mode, which plays a major role in the superconductivity enhancement [40]. The same analysis is performed to the $\beta$ mode and gives $\lambda_{\beta} \sim 0.1$. These EPC constants are mainly attributed to the interactions with electrons in FeSe films, since the electron density in STO is much lower than FeSe after electron transfer [6. The sum of the two EPC constants is $\lambda_{\alpha+\beta} \sim 0.35$, which dominantly accounts for the calculated total EPC constant about 0.4 from STO substrate [41]. The higher energy $\alpha$ mode has a stronger interaction than the $\beta$ mode, as evidenced from previous ARPES result that the 100 $\mathrm{meV}$ replica band is much clearer than the $60 \mathrm{meV}$ replica band [5. This can also be understood by the fact that the $\alpha$ mode generates stronger dipole field than the $\beta$ mode. As shown in Fig. 1 (d), all the six oxygen ions vibrate in the opposite direction with the titanium ions in the $\alpha$ mode, while the two apical oxgyen ions vibrate in the same direction with the titanium ions in the $\beta$ mode 30.

Above results give direct evidence that the electric field generated by the F-K phonon modes of STO can pene-
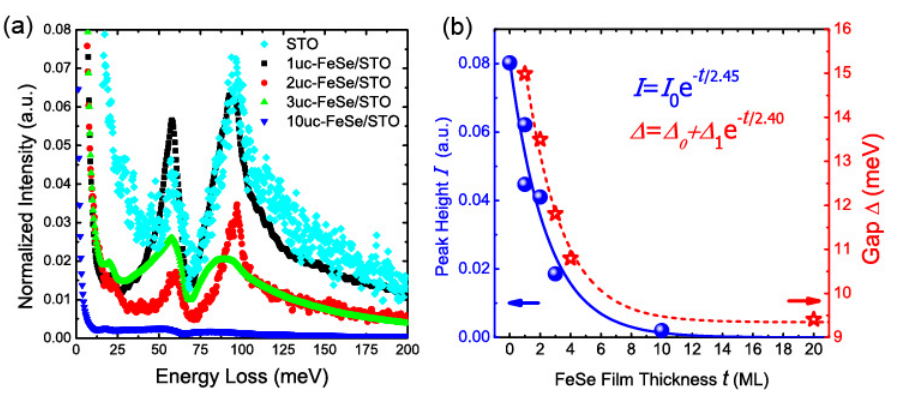

FIG. 3. (a) EDCs at the $\Gamma$ point for samples with different FeSe thickness. (b) Plot and exponential fitting of the peak height of the $\alpha$ mode as a function of the FeSe thickness (blue). Plot and exponential fitting of the superconducting gap size for the K-doped FeSe/STO as a function of the FeSe thickness (red), with data extracted from Ref. 25].

trate the FeSe films and strongly interact with the electrons in the FeSe layer. But how far can this electric field penetrate? To answer this question we performed HREELS measurements on samples with different FeSe thickness (2uc, 3uc and 10uc) to study the spacial spreading properties of the F-K modes. In Fig 3 (a) we plot the normalized EDCs at $\Gamma$ point for the samples with different FeSe thicknesses, which apparently demonstrates the decay of the $\alpha$ and $\beta$ modes with increasing FeSe thickness. An exponential fit to the normalized peak height of the $\alpha$ mode (blue dots in Fig. 3 (b)) gives the decay length of 2.5 u.c., which can be regarded as a characteristic length of the F-K mode penetration in FeSe films. A recent study of the thickness-dependent superconducting gaps of K-doped FeSe films presents very similar behavior, i.e., the gap size decreases exponentially with increasing FeSe thickness characterized by a decay length of 2.40 u.c. (red stars in Fig 3 (b)) 25]. Considering the similar carriers density at the interfacial layer [42, the striking coincidence of the two characteristic lengthes suggests that the $\alpha$ phonon of STO is closely related to the superconductivity enhancement at FeSe/STO interface. The electrons in FeSe spontaneously screen the F-K electric field and lead to the decay of the field intensity, which weakens the supconductivity enhancement. This is why the superconducting gap can only be enhanced within 3 u.c. FeSe films [43, 44].

The screening length to an external electric field by a metal is inversely proportional to the electron density of the metal 45. Raising the electron-doping level of the FeSe film, although helpful to increase the superfluid density, will inhibit the penetration length of the dipole field from STO. Therefore there is always a trade-off between the electron density and the field penetration. This is possibly the cause of gap decrease when extra electrons are doped in 1uc-FeSe/STO 43. As a result, increasing the doping level in FeSe may not be able to further enhance the superconductivity if the dipole field from 
the substrate is not penetrating effectively into the FeSe films. Searching substrates with F-K phonons that generate strong diploe field is a feasible route.

In conclusion, our HREELS study provides the microscopic understanding on the superconductivity enhancement in the FeSe/STO system. First, we determine the origination of the interfacial coupling. It is the F-K phonon modes in STO, generating long range dipole field, that strongly interact with the electrons in the FeSe layer. The mode-specific EPC constant is $\sim 0.25$ for the $\sim 92$ $\mathrm{meV}$ phonon in 1uc FeSe/STO. This interaction is closely related to the enhancement of superconductivity. Second, we determine the characteristic penetration depth of the STO F-K phonon into FeSe film. The decay of the penetrating phonon results in the thickness limit of superconductivity enhancement in FeSe/STO. Therefore ionic crystals with high energy F-K phonon modes that generate strong dipole field, for instance, oxides with high valence metal ions and long oxygen-metal bonds, should be good candidates as the substrates.

X. Zhu acknowledges helpful discussions with Prof. M. El-Batanouny, and thanks Dr. Yan Wang for pointing out the initial error in calculating the EPC constant. This work is supported by NSFC (Grant No. 11225422 and No. 11304367), MOSTC (Grant No. 2012CB921700 and No. 2015CB921000), the Strategic Priority Research Program (B) of CAS (Grant No. XDB07010100), and the External Cooperation Program of BIC, CAS (Grant No. 112111KYSB20130007).

\footnotetext{
* liliwang@mail.tsinghua.edu.cn

† xtzhu@iphy.ac.cn

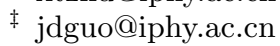

[1] Q.-Y. Wang, Z. Li, W.-H. Zhang, Z.-C. Zhang, J.-S. Zhang, W. Li, H. Ding, Y.-B. Ou, P. Deng, K. Chang, J. Wen, C.- L. Song, K. He, J.-F. Jia, S.-H. Ji, Y.-Y. Wang, L.-L. Wang, X. Chen, X.-C. Ma, and Q.-K. Xue, Chinese Physics Letters 29, 037402 (2012).

[2] W.-H. Zhang, Y. Sun, J.-S. Zhang, F.-S. Li, M.-H. Guo, Y.-F. Zhao, H.-M. Zhang, J.-P. Peng, Y. Xing, H.-C. Wang, T. Fujita, A. Hirata, Z. Li, H. Ding, C.-J. Tang, M. Wang, Q.-Y. Wang, K. He, S.-H. Ji, X. Chen, J.F. Wang, Z.-C. Xia, L. Li, Y.-Y. Wang, J. Wang, L.-L. Wang, M.-W. Chen, Q.-K. Xue, and X.- C. Ma, Chinese Physics Letters 31, 017401 (2014).

[3] W. Zhang, Z. Li, F. Li, H. Zhang, J. Peng, C. Tang, Q. Wang, K. He, X. Chen, L.Wang, X. Ma, and Q.-K. Xue, Phys. Rev. B 89, 060506 (2014).

[4] D. Liu, W. Zhang, D. Mou, J. He, Y.-B. Ou, Q.-Y. Wang, Z. Li, L. Wang, L. Zhao, S. He, et al., Nature communications 3, 931 (2012).

[5] J. Lee, F. Schmitt, R. Moore, S. Johnston, Y.-T. Cui, W. Li, M. Yi, Z. Liu, M. Hashimoto, Y. Zhang, et al., Nature 515, 245 (2014).

[6] S. Tan, Y. Zhang, M. Xia, Z. Ye, F. Chen, X. Xie, R. Peng, D. Xu, Q. Fan, H. Xu, et al., Nature materials 12,
634 (2013).

[7] J.-F. Ge, Z.-L. Liu, C. Liu, C.-L. Gao, D. Qian, Q.-K. Xue, Y. Liu, and J.-F. Jia, Nature materials 14, 285 (2015).

[8] F.-C. Hsu, J.-Y. Luo, K.-W. Yeh, T.-K. Chen, T.-W. Huang, P. M. Wu, Y.-C. Lee, Y.-L. Huang, Y.-Y. Chu, D.-C. Yan, et al., Proceedings of the National Academy of Sciences 105, 14262 (2008).

[9] S. He, J. He, W. Zhang, L. Zhao, D. Liu, X. Liu, D. Mou, Y.-B. Ou, Q.-Y. Wang, Z. Li, et al., Nature materials 12, 605 (2013).

[10] J. He, X. Liu, W. Zhang, L. Zhao, D. Liu, S. He, D. Mou, F. Li, C. Tang, Z. Li, et al., Proceedings of the National Academy of Sciences 111, 18501 (2014).

[11] Y. Zhang, L. Yang, M. Xu, Z. Ye, F. Chen, C. He, H. Xu, J. Jiang, B. Xie, J. Ying, et al., Nature materials 10, 273 (2011).

[12] L. Zhao, A. Liang, D. Yuan, Y. Hu, D. Liu, J. Huang, S. He, B. Shen, Y. Xu, X. Liu, L. Yu, G. Liu, H. Zhou, Y. Huang, X. Dong, F. Zhou, K. Liu, Z. Lu, Z. Zhao, C. Chen, Z. Xu, and X. J. Zhou, Nature Communications 7, 10608 (2016).

[13] J. Seo, B. Kim, B. Kim, J. Jeong, J. Ok, J. S. Kim, J. Denlinger, S.-K. Mo, C. Kim, and Y. Kim, Nature Communications 7, 11116 (2016).

[14] C. Wen, H. Xu, C. Chen, Z. Huang, X. Lou, Y. Pu, Q. Song, B. Xie, M. Abdel-Hafiez, D. Chareev, et al., Nature communications 7, 10840 (2016).

[15] Y. Miyata, K. Nakayama, K. Sugawara, T. Sato, and T. Takahashi, Nature materials 14, 775 (2015).

[16] C.-L. Song, H.-M. Zhang, Y. Zhong, X.-P. Hu, S.-H. Ji, L. Wang, K. He, X.-C. Ma, and Q.-K. Xue, Phys. Rev. Lett. 116, 157001 (2016).

[17] J. Shiogai, Y. Ito, T. Mitsuhashi, T. Nojima, and A. Tsukazaki, Nature Physics 12, 42 (2016).

[18] B. Lei, J. H. Cui, Z. J. Xiang, C. Shang, N. Z. Wang, G. J. Ye, X. G. Luo, T. Wu, Z. Sun, and X. H. Chen, Phys. Rev. Lett. 116, 077002 (2016).

[19] K. Hanzawa, H. Sato, H. Hiramatsu, T. Kamiya, and H. Hosono, Proceedings of the National Academy of Sciences 113, 3986 (2016).

[20] R. Peng, X. P. Shen, X. Xie, H. C. Xu, S. Y. Tan, M. Xia, T. Zhang, H. Y. Cao, X. G. Gong, J. P. Hu, B. P. Xie, and D. L. Feng, Phys. Rev. Lett. 112, 107001 (2014).

[21] R. Peng, H. Xu, S. Tan, H. Cao, M. Xia, X. Shen, Z. Huang, C. Wen, Q. Song, T. Zhang, et al., Nature communications 5, 5044 (2014).

[22] P. Zhang, X.-L. Peng, T. Qian, P. Richard, X. Shi, J.-Z. Ma, B.-B. Fu, Y.-L. Guo, Z. Han, S. Wang, et al., arXiv preprint arXiv:1512.01949 (2015).

[23] G. Zhou, D. Zhang, C. Liu, C. Tang, X. Wang, Z. Li, C. Song, S. Ji, K. He, L. Wang, X. Ma, and Q.-K. Xue, Applied Physics Letters 108, 202603 (2016).

[24] C.-L. Song, Y.-L.Wang, P. Cheng, Y.-P. Jiang, W. Li, T. Zhang, Z. Li, K. He, L. Wang, J.-F. Jia, H.-H. Hung, C. Wu, X. Ma, X. Chen, and Q.-K. Xue, Science 332, 1410 (2011).

[25] W. H. Zhang, X. Liu, C. H. P. Wen, R. Peng, S. Y. Tan, B. P. Xie, T. Zhang, and D. L. Feng, Nano Letters 16, 1969 (2016).

[26] Y.-T. Cui, R. G. Moore, A.-M. Zhang, Y. Tian, J. J. Lee, F. T. Schmitt, W.-H. Zhang, W. Li, M. Yi, Z.-K. Liu, M. Hashimoto, Y. Zhang, D.-H. Lu, T. P. Devereaux, L.-L. Wang, X.-C. Ma, Q.-M. Zhang, Q.-K. Xue, D.-H. Lee, 
and Z.-X. Shen, Phys. Rev. Lett. 114, 037002 (2015).

[27] Y. C. Tian, W. H. Zhang, F. S. Li, Y. L. Wu, Q. Wu, F. Sun, G. Y. Zhou, L. Wang, X. Ma, Q.-K. Xue, and J. Zhao, Phys. Rev. Lett. 116, 107001 (2016).

[28] R. Fuchs and K. L. Kliewer, Phys. Rev. 140, A2076 (1965).

[29] see Supplementary Material for details.

[30] H. Vogt, Phys. Rev. B 38, 5699 (1988).

[31] X. Zhu, Y. Cao, S. Zhang, X. Jia, Q. Guo, F. Yang, L. Zhu, J. Zhang, E. Plummer, and J. Guo, Review of Scientific Instruments 86, 083902 (2015).

[32] A. D. Baden, P. A. Cox, R. G. Egdell, A. F. Orchard, and R. J. D. Willmer, Journal of Physics C: Solid State Physics 14, L1081 (1981).

[33] H. Ibach and D. L. Mills, Electron energy loss spectroscopy and surface vibrations (Academic press, 1982).

[34] J. L. Servoin, Y. Luspin, and F. Gervais, Phys. Rev. B 22, 5501 (1980).

[35] C. Perry, J. H. Fertel, and T. McNelly, The Journal of Chemical Physics 47, 1619 (1967).

[36] G. Grimvall, The electron-phonon interaction in metals, Vol. 8 (North-Holland Amsterdam, 1981).

[37] J. Menéndez and M. Cardona, Phys. Rev. B 29, 2051 (1984).

[38] P. B. Allen, Solid State Communications 14, 937 (1974).

[39] P. Allen, in Dynamical properties of solids. Volume 3 (1980) p. 95.

[40] Y. Wang, A. Linscheid, T. Berlijn, and S. Johnston, Phys. Rev. B 93, 134513 (2016).

[41] Y. Zhou and A. J. Millis, Phys. Rev. B 93, 224506 (2016).

[42] Z. R. Ye, C. F. Zhang, H. L. Ning, W. Li, L. Chen, T. Jia, M. Hashimoto, D. H. Lu, Z.-X. Shen, and Y. Zhang, arXiv preprint arXiv:1512.02526 (2015).

[43] C. Tang, D. Zhang, Y. Zang, C. Liu, G. Zhou, Z. Li, C. Zheng, X. Hu, C. Song, S. Ji, K. He, X. Chen, L. Wang, X. Ma, and Q.-K. Xue, Phys. Rev. B 92, 180507 (2015).

[44] C. Tang, C. Liu, G. Zhou, F. Li, H. Ding, Z. Li, D. Zhang, Z. Li, C. Song, S. Ji, K. He, L. Wang, X. Ma, and Q.-K. Xue, Phys. Rev. B 93, 020507 (2016).

[45] A. K. Theophilou, Journal of Physics F: Metal Physics 2, 1124 (1972). 


\section{Supplemental Materials}

\section{SAMPLE PREPARATION AND CHARACTERIZATION}

$\mathrm{Nb}$-doped $\mathrm{STO}(001)$ substrate was first degassed overnight at $600{ }^{\circ} \mathrm{C}$ in ultra high vacuum (UHV), and then annealed at $950{ }^{\circ} \mathrm{C}$ for 40 minutes. FeSe films were grown by co-depositing high-purity Fe (99.99\%) and Se (99.99+\%) with a flux ratio of $\sim 1: 20$ onto the STO substrate held at $400{ }^{\circ} \mathrm{C}$. The as-prepared samples were post-annealed at $470{ }^{\circ} \mathrm{C}$ for 5 hours in UHV to make the first layer FeSe superconducting. The in situ scanning tunneling microscopy (STM) measurements were performed to confirm the sample quality. Fig. S1 (a) gives the topographic image which shows large surface steps $(\sim 100 \mathrm{~nm})$ of $1 \mathrm{uc}-\mathrm{FeSe} / \mathrm{STO}$. The dark strips correspond to domain boundaries in FeSe films due to the strain between STO substrate and FeSe single layer. The lattice constant of the FeSe layer is 3.8 $\AA$ as shown in Fig. S1 (b). The scanning tunneling spectroscopy (STS) measurement in Fig. S1 (c) indicates the superconducting gap $(\Delta)$ of annealed 1uc-FeSe/STO is $\sim 20 \mathrm{meV}$. The sample was protected by a thick amorphous Se capping layer deposited at $110 \mathrm{~K}$ and transformed to the 2D-HREELS system.

(a)

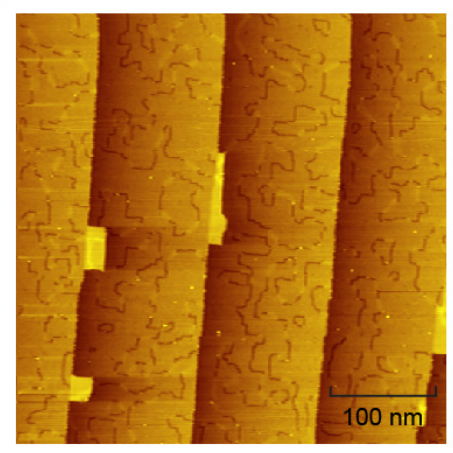

(b)

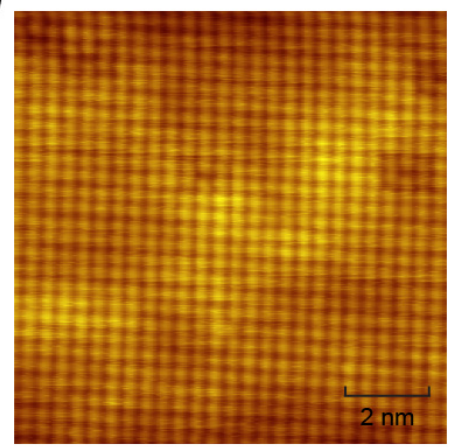

(c)

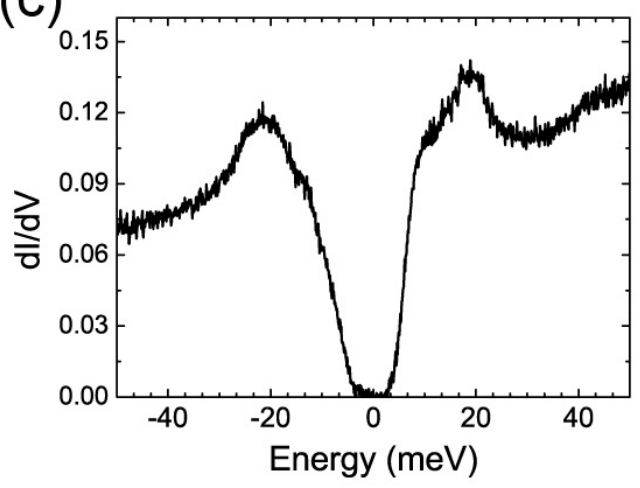

FIG. S1. (a) STM topography of 1 uc-FeSe/STO sample (Image size: $400 \times 400 \mathrm{~nm}$, sample bias $V_{b}=5.0 \mathrm{~V}$, tunneling current $I_{t}=50 \mathrm{pA}$ ). (b) Atomically resolved STM topography of 1uc-FeSe/STO sample (Image size: $10 \times 10 \mathrm{~nm}$, sample bias $V_{b}=0.4$ $\mathrm{V}$, tunneling current $I_{t}=100 \mathrm{pA}$ ). (c) dI/dV spectrum of 1uc-FeSe/STO. All Spectra were taken at $4.2 \mathrm{~K}$.

In the 2D-HREELS system, the sample was annealed at $450{ }^{\circ} \mathrm{C}$ to remove the capping layer. ARPES was performed to detect the electronic structure (Fig. S2 (a)) and supercongucting gap (Fig. S2 (d)) to confirm the existence of superconducting state in the sample. ARPES spectra were scanned along the $\Gamma$ to $M$ direction, corresponding to the horizontal direction in the LEED pattern in Fig. S2 (c). A parabolic electron band can be clearly observed in the second derivative spectrum (Fig. S2 (b)). Temperature-dependent symmetrized EDCs at $k_{F}$ reveal the superconducting gap at $35 \mathrm{~K}$ is $\sim 20 \mathrm{meV}$ and closes between $63 \mathrm{~K}$ and $73 \mathrm{~K}$ (As shown in Fig. S2.d)). 

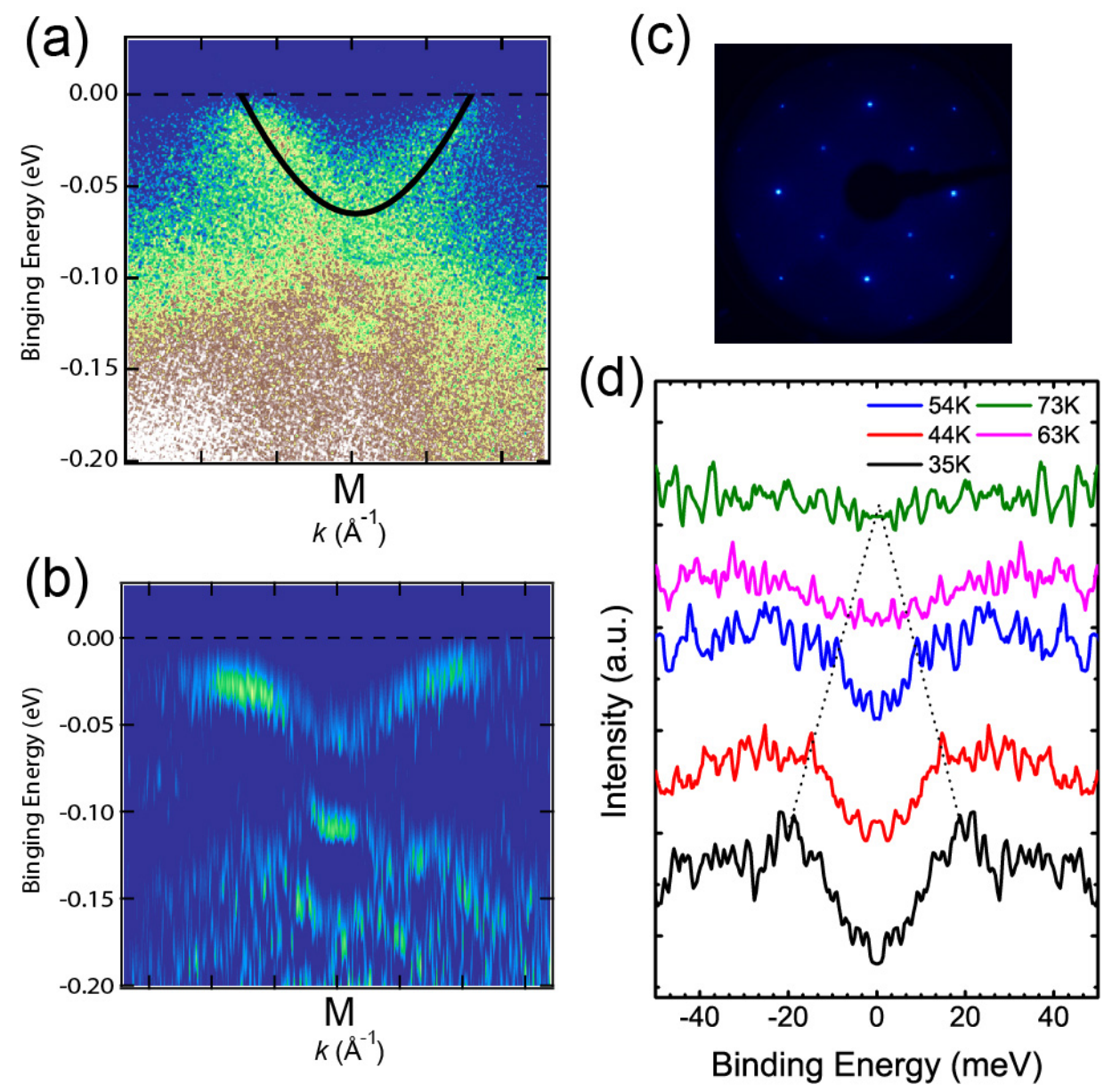

FIG. S2. (a) ARPES spectrum of 1uc-FeSe/STO sample taken at $35 \mathrm{~K} . k$ refers to the momentum along the $\Gamma-M$ direction and centered at $M$. The black line represents the band fitting by a tight binding model. (b) Second derivative spectrum for (a). (c) LEED pattern of 1uc-FeSe/STO sample taken at $35 \mathrm{~K}$ with $80 \mathrm{eV}$ electron beam energy. (d) Plot of the evolution of the symmetrized EDCs at $k_{F}$ as a function of temperature, which shows the gap closes between $63 \mathrm{~K}$ and $73 \mathrm{~K}$.

\section{HREELS EXPERIMENTS AND PHONON MODE ASSIGNMENT}

The HREELS experiments were performed on bare STO and on thin FeSe films grown on STO with different thickness (1uc, 2uc, 3uc and $10 \mathrm{uc}$ ). The $\mathrm{STO}(001)$ sample was annealed at $900{ }^{\circ} \mathrm{C}$ for 1 hour before the $\mathrm{HREELS}$ measurements. Most of the HREELS measurements were performed with an incident beam energy of 50 eV unless stated otherwise.

Fig. S3 (a) shows the 2D energy-momentum mapping of the bare STO(001) sample. There are only two energy loss features at 60 ( $\beta$ mode) and $97 \mathrm{meV}$ ( $\alpha$ mode), corresponding to the F-K surface phonon modes of STO [S1], which is consistent with previous HREELS measurements [S2, S3. However, for the FeSe/STO samples, 5 phonon modes are observed. Both theoretical [S4] and experimental [S5, S6] studies of FeSe have shown that the energy of all FeSe phonon modes should be smaller than $40 \mathrm{meV}$. Therefore the $\alpha(92 \mathrm{meV})$ and $\beta$ (60 meV) modes observed from FeSe/STO samples should be the F-K modes from STO substrate. $\eta, \sigma$ and $\zeta$ modes, which are absent in bare STO sample (Fig. S3 (a)), are phonon modes of FeSe lattice. This assignment can also be verified by comparing the HREELS results of FeSe films with different thickness. Fig. SS3 (b), (c) and (d) show the 2D-HREELS mapping of 1uc-FeSe/STO, 3uc-FeSe/STO and 10uc-FeSe/STO, respectively. Clearly the F-K modes of STO ( $\alpha$ and $\beta$ ) gradually become invisible with increasing FeSe thickness. In contrast, the $\eta, \gamma$ and $\zeta$ modes become clearer. Actually this is one of the key reasons why we assign $\eta, \gamma$ and $\zeta$ to be the phonon modes from FeSe, rather than from STO. $\beta$ and $\alpha$ phonon modes observed in FeSe/STO reveals that the electric field generated by F-K phonon of STO can penetrate into the FeSe films and decay exponentially with thickness increasing (Fig. 3 in the main paper). 
(a) STO

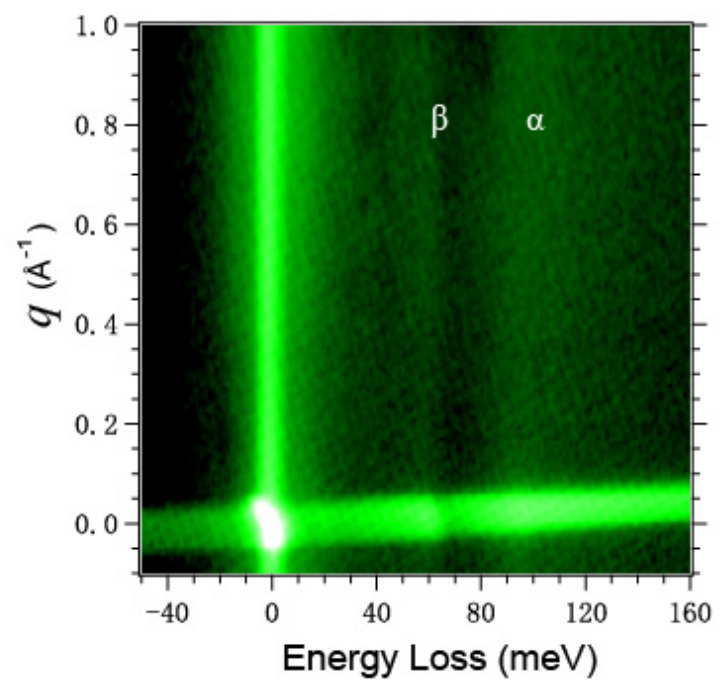

(c) 3uc-FeSe/STO

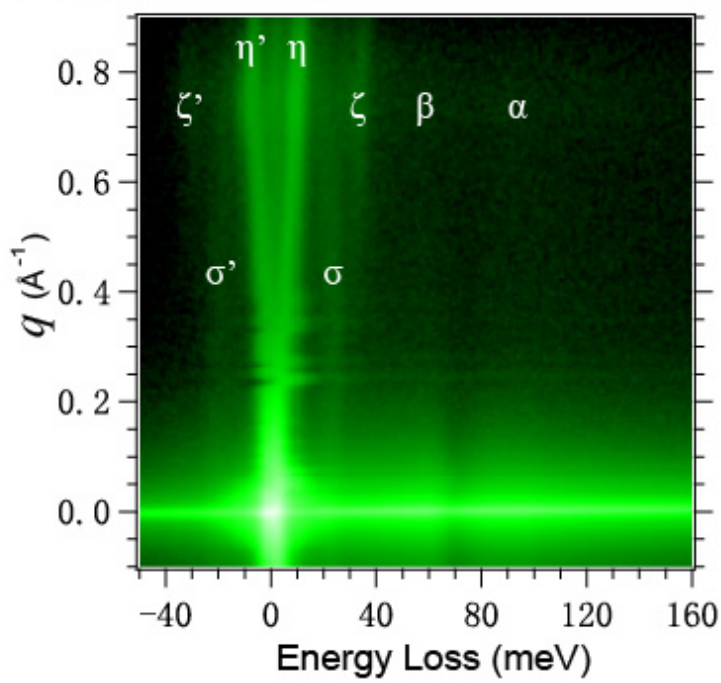

(b) 1uc-FeSe/STO

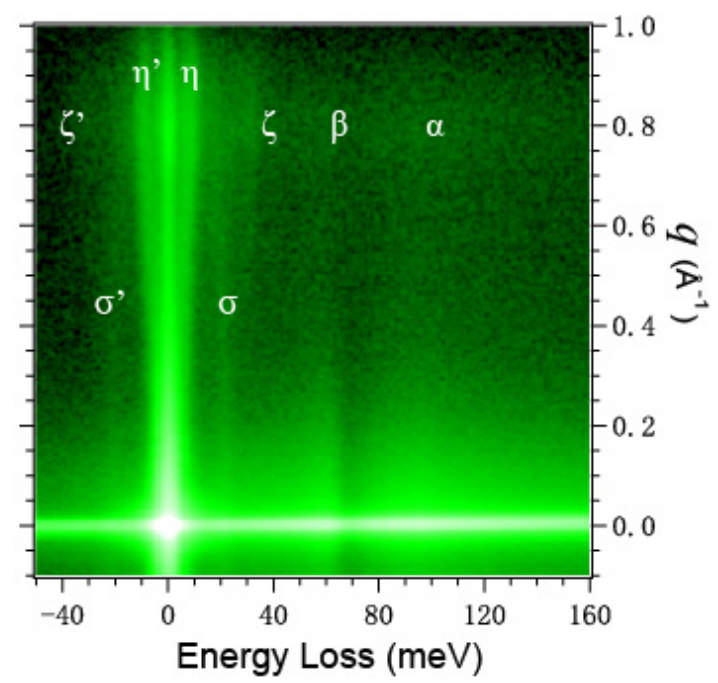

(d) $10 \mathrm{uc}-\mathrm{FeSe} / \mathrm{STO}$

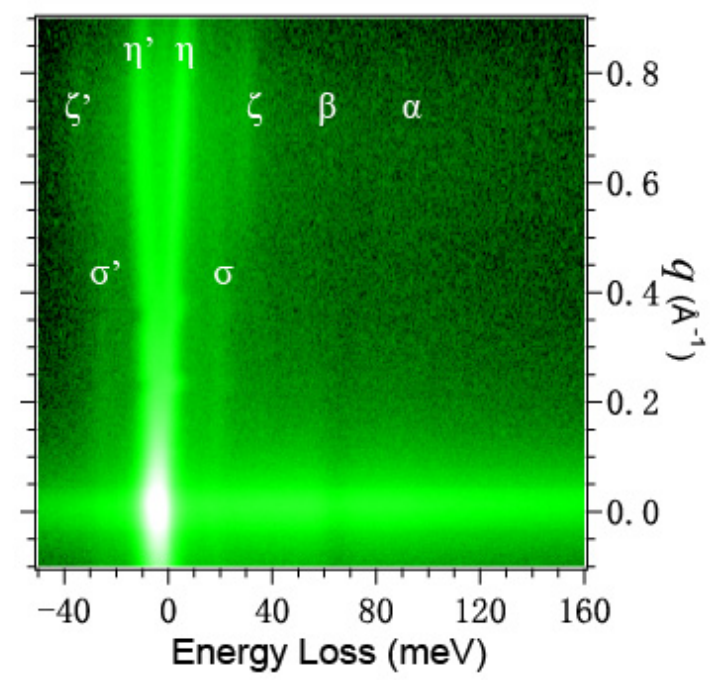

FIG. S3. 2D HREELS energy-momentum mapping along the $\Gamma$-X direction for (a) STO(001), (b) 1uc-FeSe/STO, (c) 3ucFeSe/STO and (d) 10uc-FeSe/STO. The incident electron energy is $120 \mathrm{eV}$ for (a) and $50 \mathrm{eV}$ for (b)-(c). Greek letters are used to label the energy loss features. The positive energy loss features are labeled by $\alpha, \beta, \sigma, \zeta$, and $\eta$, respectively. The corresponding negative energy loss features correspond to their anti-Stokes peaks, which are labeled by $\sigma^{\prime}, \zeta^{\prime}$, etc.

The detailed assignment of the FeSe phonon modes are based on the selection rule of HREELS [S7] and previous Raman experiments [S8, S9. $\eta$ mode is an acoustic phonon branch, $\sigma$ mode is $A_{1 g}$ optical phonon, and $\zeta$ should be $E_{g}$ or $A_{2 u}$ optical phonon in FeSe.

\section{CALCULATION OF THE EPC CONSTANT}

Tight binding model and Density of State

The Density of state (DOS) can be calculated through the electron band dispersion by a simple tight binding model:

$$
\epsilon_{e, h}(k)=-2 t_{e, h} \cos (k a)-\mu_{e, h}
$$


with $\mathrm{t}_{e}=125 \mathrm{meV}, \mathrm{t}_{h}=-30 \mathrm{meV}, \mu_{e}=-185 \mathrm{meV}$ and $\mu_{h}=175 \mathrm{meV}$ S10. The fitting of the band was shown in Fig. S2 (a).

The area in $\mathrm{k}$-space for $2 \mathrm{D}$ is expressed as:

$$
\Omega(E)=\pi k^{2} .
$$

Therefore, the density of state (DOS) for both spin direction is:

$$
N(E)=\frac{2}{\left(\frac{2 \pi}{a}\right)^{2}} \frac{d \Omega(E)}{d E}=\frac{a^{2}}{2 \pi} \frac{k}{\frac{d \epsilon_{e}}{d k}}=\frac{a}{2 \pi t_{e}} \frac{k}{\sin (k a)}
$$

For 1uc-FeSe/STO, a $\sim 3.8 \AA$, and Fermi wave number $k_{F}=0.19 \AA^{-1}$. Therefore, $N\left(E_{F}\right)=1.4 \times 10^{-3}(\mathrm{meV})^{-1}$.

\section{Extracting EPC-induced phonon line widths from experimental results: anharmonic phonon decay}

The energy for a specific phonon branch $\nu$ at temperature $T$ and momentum q can be written as a complex form S11.

$$
\widetilde{\omega}(\mathbf{q}, \nu, T)=\operatorname{Re}[\widetilde{\omega}(\mathbf{q}, \nu, T)]-i \operatorname{Im}[\widetilde{\omega}(\mathbf{q}, \nu, T)],
$$

with the energy as the real part

$$
\operatorname{Re}[\widetilde{\omega}(\mathbf{q}, \nu, T)]=\omega_{0}(\mathbf{q}, \nu)+\Delta \omega_{V}(\mathbf{q}, \nu ; T)+\Delta \omega_{p p}(\mathbf{q}, \nu ; T),
$$

and the line width (half width at half maximum) as the imaginary part

$$
\operatorname{Im}[\widetilde{\omega}(\mathbf{q}, \nu, T)]=\gamma_{e p}(\mathbf{q}, \nu)+\gamma_{p p}(\mathbf{q}, \nu ; \mathbf{T}),
$$

where $\omega_{0}(\mathbf{q}, \nu)$ is the harmonic phonon energy at $T=0 \mathrm{~K}$ including the $T$-independent EPC contribution, $\Delta \omega_{V}(\mathbf{q}, \nu ; \mathbf{T})$ is the energy shift due to the change in the volume, and $\Delta \omega_{p p}(\mathbf{q}, \nu ; \mathbf{T})$ is the energy shift due to the anharmonic phonon-phonon interactions. The imaginary term $-i \operatorname{Im}[\widetilde{\omega}(\mathbf{q}, \nu, T)]$ describes the damping of the phonons, with contributions from both $\operatorname{EPC} \gamma_{e p}(\mathbf{q}, \nu ; \mathbf{T})$ and anharmonicity $\gamma_{p p}(\mathbf{q}, \nu ; \mathbf{T})$.

To obtain the EPC constant from Allen's formula, the EPC-induced line width $\gamma_{e p}$ is a prerequisite quantity. Thus extracting the contribution of EPC from measured phonon line widths is necessary. Up to now, however, it is still a challenge in practice to directly obtain the pure EPC induced phonon line widths from experiments. This is mainly because the measured phonon line widths will probably also contain additional contributions from anharmonic phonon-phonon interaction. Only when the temperature-dependent anharmonic phonon-phonon interaction is correctly deducted, can the EPC-induced line width $\gamma_{e p}$ be determined and $\lambda$ be calculated.

Here we consider three different phonon decay channels to model the anharmonic effect:

(1) An optical phonon with energy $\hbar \omega_{0}$ decays into two acoustic phonons with energy $\hbar \omega_{0} / 2$, which is a three phonon decay process $\underline{\mathbf{S 1 2}}$. In this case the temperature-dependent phonon energy and line width can be expressed as:

$$
\begin{gathered}
\hbar \omega(T)=\hbar \omega_{0}+\hbar \omega_{a}\left(1+\frac{2}{e^{\hbar \omega_{0} / 2 k_{B} T}-1}\right) \\
\gamma(T)=\gamma_{e p}\left(1+\frac{2}{e^{\hbar \omega_{0} / 2 k_{B} T}-1}\right)
\end{gathered}
$$

(2) An optical phonon mode with energy $\hbar \omega_{0}$ decays into three acoustic phonon modes with energy $\hbar \omega_{0} / 3$, which is a four phonon decay process [S13]. In this case the temperature-dependent phonon energy and line width can be expressed as:

$$
\begin{gathered}
\hbar \omega(T)=\hbar \omega_{0}+\hbar \omega_{a}\left(1+\frac{2}{e^{\hbar \omega_{0} / 2 k_{B} T}-1}\right)+\hbar \omega_{b}\left[1+\frac{3}{e^{\hbar \omega_{0} / 3 k_{B} T}-1}+\frac{3}{\left(e^{\hbar \omega_{0} / 3 k_{B} T}-1\right)^{2}}\right] \\
\gamma(T)=\gamma_{a}\left(1+\frac{2}{e^{\hbar \omega_{0} / 2 k_{B} T}-1}\right)+\gamma_{b}\left[1+\frac{3}{e^{\hbar \omega_{0} / 3 k_{B} T}-1}+\frac{3}{\left(e^{\hbar \omega_{0} / 3 k_{B} T}-1\right)^{2}}\right]
\end{gathered}
$$


(3) An optical phonon mode with energy $\hbar \omega_{0}$ decays into another two optical phonon modes with lower eneryies $\hbar \omega_{1}$ and $\hbar \omega_{1}$ S14. In this case the temperature-dependent phonon energy and line width can be expressed as:

$$
\begin{gathered}
\hbar \omega(T)=\hbar \omega_{0}+\hbar \omega_{a}\left(1+\frac{1}{e^{\hbar \omega_{1} / k_{B} T}-1}+\frac{1}{e^{\hbar \omega_{2} / k_{B} T}-1}\right) \\
\gamma(T)=\gamma_{e p}\left(1+\frac{1}{e^{\hbar \omega_{1} / k_{B} T}-1}+\frac{1}{e^{\hbar \omega_{2} / k_{B} T}-1}\right)
\end{gathered}
$$

(a)

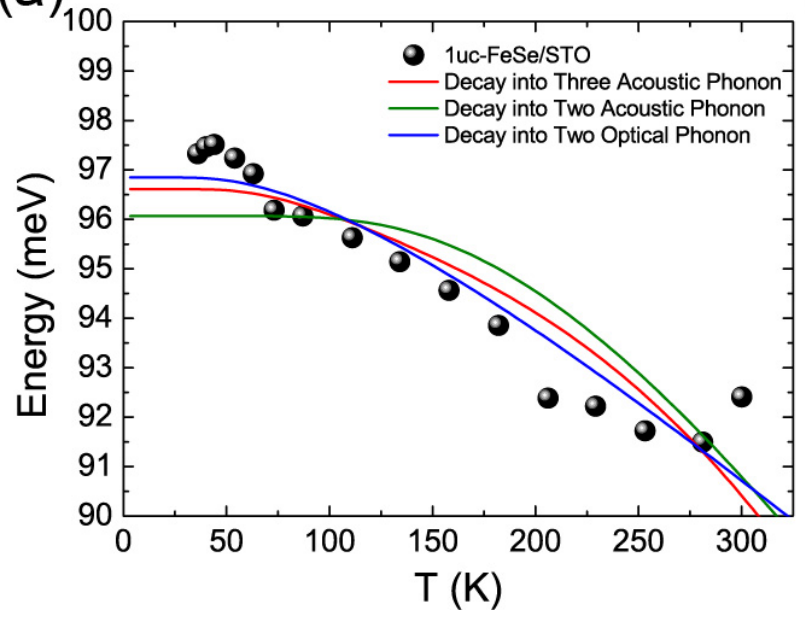

(b)

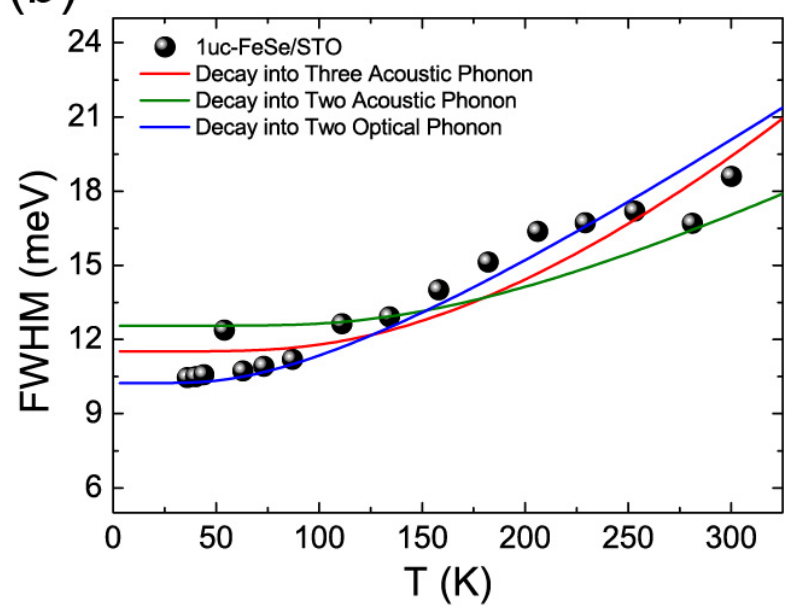

FIG. S4. The temperature dependence of the energy (a) and full width at half maximum (FWHM) (b) of the $\alpha$ phonon branch of the 1uc-FeSe/STO sample. The dots are experimental data obtained by fitting the EDCs from the HREELS spectra at the $\Gamma$ point with Gaussian peaks, and the solid lines stands for anharmonic decay fits of the data (red: $\alpha$ phonon branch decay into three acoustic phonon; green: $\alpha$ phonon branch decay into two acoustic phonon; blue: $\alpha$ phonon branch decay into two optical phonon). The line width in (b) was obtained by deconvoluting the measured phonon line width with the elastic peak width, to remove the contributions from the instrumentation broadening and surface roughness.

The fitting result of these three decay models for the $\alpha$ mode are exhibited in Fig. S4 (a) and (b). The third model, an optical phonon $(92 \mathrm{meV})$ decays into two optical phonons (60 and $32 \mathrm{meV}$ phonon branch), provides best fitting results for $\alpha$ mode.

Therefore we can extract $\gamma_{e p}$ for $\alpha$ mode through above anharmonic phonon decay model. At $T=0$, there is no phonon-phonon interaction, thus $\hbar \omega(T=0)$ and $\gamma(T=0)$ are the energy and line width with EPC only. As a result we have extracted the EPC induced line width $\gamma_{e p}(\alpha)=\gamma(T=0) \cong 5.1 \mathrm{meV}$. With $\gamma_{e p}$ and electron DOS, the EPC constant for $\alpha$ mode is deduced from Allen's formula: $\lambda_{\alpha} \sim 0.27$.

\section{Extracting EPC-induced phonon line widths from experimental results: An approach of Kramers-Kronig relation}

Some other indirect methods have also been tried to extract the EPC-induced phonon line widths from experimental measurements. The renormalization of the phonon energies and the line widths are related by a Kramers-Kronig relation (or sometimes called Hilbert transformation). If the unharmonicity is corrected (or showed to be negligible), the EPC-induced line widths can be easily extracted. Such an example is shown in a recent study of the electronphonon coupling on the surface of topological insulators $\mathrm{S15}$. Here we will employ a similar approach to extract the EPC-induced line width in FeSe/STO system and compare the results with anharmonic phonon decay method.

Again we focus on the $\alpha$ phonon branch that almost has no dispersion, then the phonon energy is nearly qindependent. Moreover, since the thickness of FeSe film is only 1 u.c. (about $0.5 \mathrm{~nm}$ ), thus the energy shift due to the volume change $\Delta \omega_{V}(\alpha ; T)$ is mainly from the STO substrate. Here we employ a simple linear fitting to both the 
STO and 1uc-FeSe/STO data, as shown in Fig. S5 (a), and obtain:

$$
\begin{aligned}
& \Delta \omega_{V}(\alpha ; T)+\Delta \omega_{p p, S T O}(\alpha ; T)=0.008 T \\
& \Delta \omega_{V}(\alpha ; T)+\Delta \omega_{p p, S T O}(\alpha ; T)+\Delta \omega_{p p, F e S e}(\alpha ; T)=-0.024 T
\end{aligned}
$$

So the energy shift due to the unharmornic phonon-phonon interactions from the FeSe film is:

$$
\Delta \omega_{p p, F e S e}(\alpha ; T)=-0.032 T
$$

This energy shift can be rewritten in terms of energy (or frequency) as:

$$
\Delta \omega_{p p}(\alpha ; T, \omega)=\frac{0.032 T}{98.26-0.024 T} \omega
$$

(a)

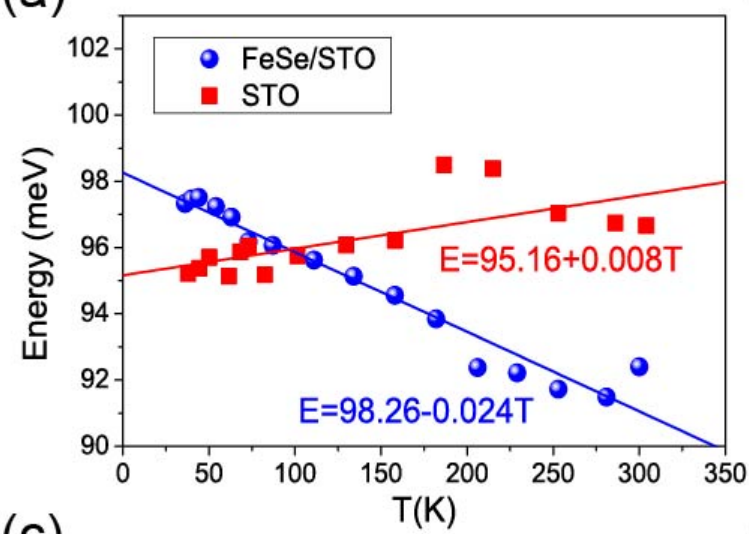

(c)

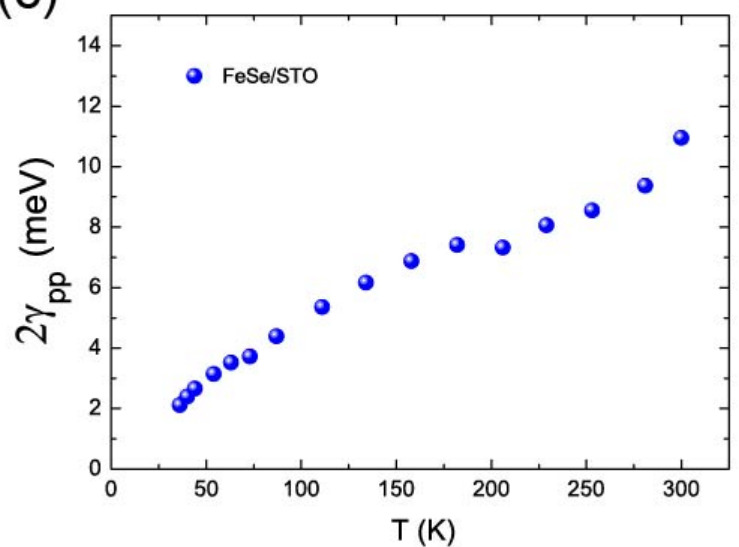

(b)

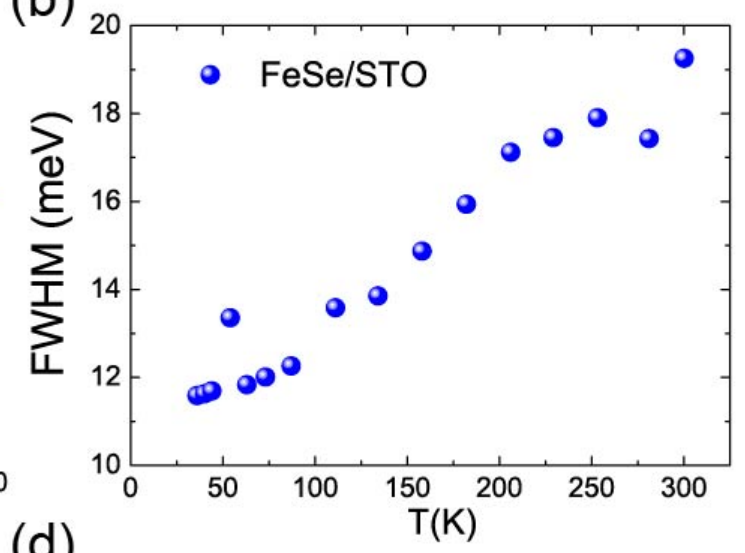

(d)

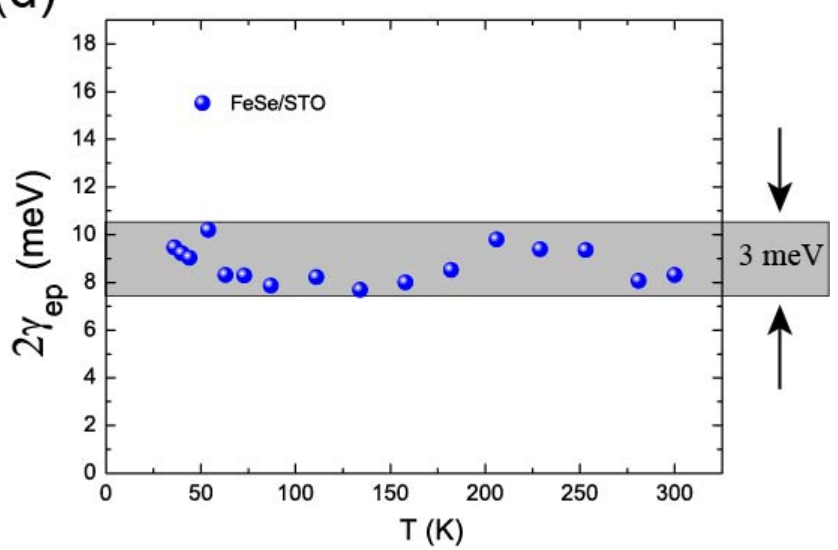

FIG. S5. The temperature dependence of the energy (a) and full width at half maximum (FWHM) (b) of the $\alpha$ phonon mode, blue for 1uc-FeSe/STO, and red for bare STO. The dots are experimental data obtained by fitting the EDCs from the HREELS spectra at the $\Gamma$ point with Gaussian peaks, and the solid lines stands for linear fits of the data. The line width in (b) was obtained by deconvoluting the measured phonon line width with the elastic peak width, to remove the contributions from the instrumentation broadening and surface roughness. The line width contribution from anharmonic phonon-phonon interactions (c) and from EPC (d) of the $\alpha$ mode. The gray band in (d) represents the energy resolution of our facility.

Then the Kramers-Kronig relation

$$
\gamma_{p p}(\alpha ; T, \omega)=\frac{2}{\pi} \int_{0}^{\infty} \frac{\omega \Delta \omega_{p p}\left(\alpha ; T, \omega^{\prime}\right)}{\omega^{2}-\omega^{\prime 2}} d \omega^{\prime}
$$

can be used to calculate the line width contribution from unharmonic phonon-phonon interactions. In real calculation, instead of using infinity, we used the highest phonon energy $\omega_{\max }=100 \mathrm{meV}$ as a cutoff energy for the upper limit 
of the integral. The results of $\gamma_{p p}$ are plotted in Fig S5 (c). Then the EPC-induced line width $\gamma_{e p}$ is obtained by subtracting $\gamma_{p p}$ from the measured width in Fig. S5 (b). As expected, the resulting $\gamma_{e p}$ is very weakly temperaturedependent, especially when our instrument resolution of about $3 \mathrm{meV}$ is taken into account. Consequently we obtain an average EPC-induced line width $\overline{\gamma_{e p}(\alpha)}=4.5 \pm 0.5 \mathrm{meV}$, yielding a EPC constant $\lambda_{\alpha} \sim 0.24 \pm 0.03$.

So we have obtained similar $\gamma_{e p}$ as well as $\lambda_{\alpha}$ from two independent methods, which indicates the feasibility and reliability of our EPC constant calculation. Finally we adopt an average value of the EPC constant for the $\alpha$ mode: $\lambda_{\alpha} \sim 0.25$.

\section{Extracting EPC-induced phonon line widths of $\beta$ branch}

The aforementioned approaches are also applied to the $\beta$ mode. The same anharmonic phonon decay models are used to fit the temperature-dependent energy and line width as well, with results plotted in Fig. S6 (a) and (b). Similarly, the third model, an optical phonon $(\beta \sim 60 \mathrm{meV})$ decays into another two optical phonons $(\zeta \sim 32 \mathrm{meV}$, and $\sigma \sim 25 \mathrm{meV}$ at zone boundary), provides the best fitting results.

The EPC-induced line width $\gamma_{e p}$ of $\beta$ mode is extracted from Fig. S6 (b): $\gamma_{e p}(\beta) \sim 0.8$ meV. And using Allen's formula, the corresponding EPC constant is obtained: $\lambda_{\beta} \sim 0.1$. This phonon mode had also been observed as a replica band in ARPES spectra but can not be used to calculate EPC constant due to the weak intensity $\mathrm{S10}$. Whereas, HREELS provides a excellent technique to detect surface elementary excitations and enables us to acquire coupling constant between phonons from STO substrate and electrons in FeSe films. The electric field of the $\beta$ mode can also penetrate FeSe films. An exponential fit to the normalized peak height of the $\beta$ mode, as a function of the FeSe thickness, yields a decay length of $3.68 \pm 2.69$ u.c. (Fig. S6 (c)), which is similar to the penetration depth of the $\alpha$ mode. The penetration of the $\beta$ mode actually provides one of the phonon decay channels for the $\alpha$ mode in FeSe films.

Comparing with the $\alpha$ phonon mode, in which the growth of single layer FeSe strengthens the anharmonic phonon decay, the $\beta$ mode exhibits opposite tendency. The anharmonicity of the $\beta$ mode of bare STO is stronger than that of 1uc-FeSe/STO, since the decay of the $\beta$ mode energy as a function of temperature of bare STO is stronger (shown in Fig. S6 (a)). Therefore, the anharmonic decay of the $\beta$ mode is much smaller than that of the $\alpha$ mode, which is possibly an important reason why $\lambda_{\beta}$ is smaller than $\lambda_{\alpha}$.

(a)

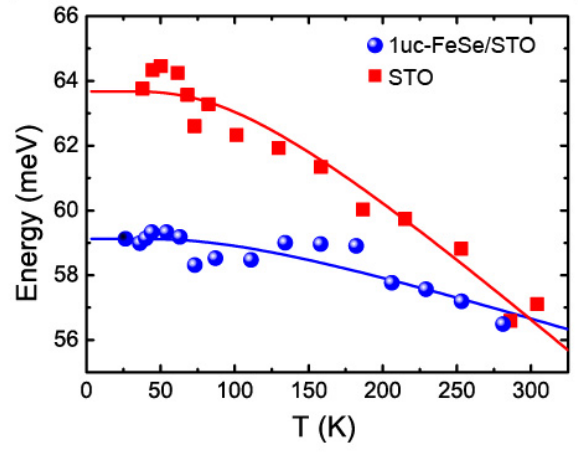

(b)

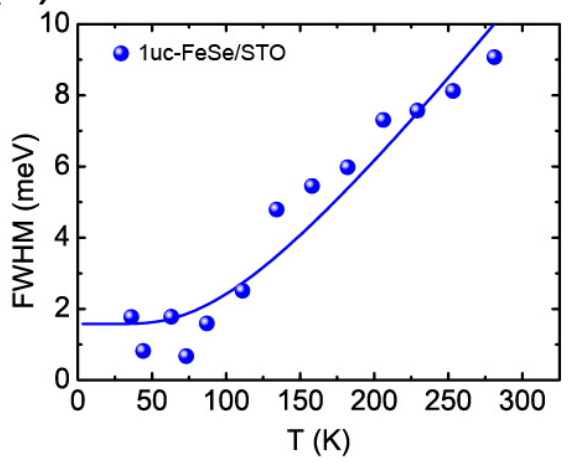

(c)

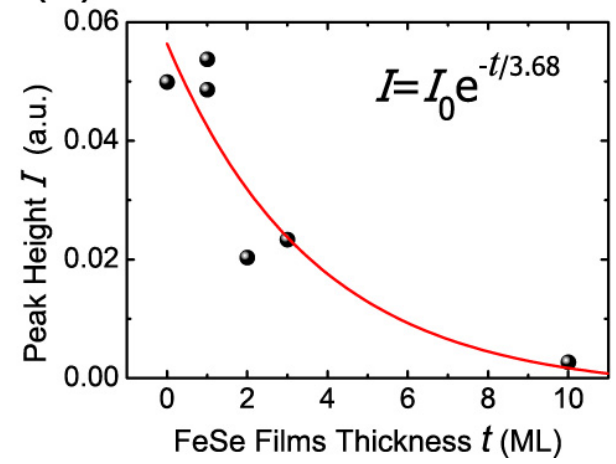

FIG. S6. The temperature dependence of the energy (a) and full width at half maximum (FWHM) (b) of the $\beta$ phonon branch. The dots are experimental data obtained by fitting the EDCs from the HREELS spectra at the $\Gamma$ point with Gaussian peaks, and the solid lines stand for an anharmonic phonon fitting of the data (red: STO; blue: 1uc-FeSe/STO). The line width in (b) was obtained by deconvoluting the measured phonon line width with the elastic peak width, to remove the contributions from the instrumentation broadening and surface roughness. (c) Plot and exponential fitting of the peak height of the $\beta$ mode as a function of the FeSe thickness.

* liliwang@mail.tsinghua.edu.cn

$\dagger$ xtzhu@iphy.ac.cn 
$\ddagger$ jdguo@iphy.ac.cn

[S1] R. Fuchs and K. L. Kliewer, Phys. Rev. 140, A2076 (1965).

[S2] T. Conard, L. Philippe, P. Thiry, P. Lambin, and R. Caudano, Surface Science 287, 382 (1993).

[S3] A. D. Baden, P. A. Cox, R. G. Egdell, A. F. Orchard, and R. J. D. Willmer, Journal of Physics C: Solid State Physics 14, L1081 (1981).

[S4] A. Subedi, L. Zhang, D. J. Singh, and M. H. Du, Phys. Rev. B 78, 134514 (2008).

[S5] D. Phelan, J. N. Millican, E. L. Thomas, J. B. Leão, Y. Qiu, and R. Paul, Phys. Rev. B 79, 014519 (2009).

[S6] V. Ksenofontov, G. Wortmann, A. I. Chumakov, T. Gasi, S. Medvedev, T. M. McQueen, R. J. Cava, and C. Felser, Phys. Rev. B 81, 184510 (2010).

[S7] F. de Juan, A. Politano, G. Chiarello, and H. A. Fertig, Carbon 85, 225 (2015).

[S8] K. Okazaki, S. Sugai, S. Niitaka, and H. Takagi, Phys. Rev. B 83, 035103 (2011).

[S9] P. Kumar, A. Kumar, S. Saha, D. Muthu, J. Prakash, S. Patnaik, U. Waghmare, A. Ganguli, and A. Sood, Solid State Communications 150, 557 (2010).

[S10] J. Lee, F. Schmitt, R. Moore, S. Johnston, Y.-T. Cui, W. Li, M. Yi, Z. Liu, M. Hashimoto, Y. Zhang, et al., Nature 515, 245 (2014).

[S11] G. Grimvall, The electron-phonon interaction in metals, Vol. 8 (North-Holland Amsterdam, 1981).

[S12] T. R. Hart, R. L. Aggarwal, and B. Lax, Phys. Rev. B 1, 638 (1970).

[S13] M. Balkanski, R. F. Wallis, and E. Haro, Phys. Rev. B 28, 1928 (1983).

[S14] J. Menéndez and M. Cardona, Phys. Rev. B 29, 2051 (1984).

[S15] X. Zhu, L. Santos, C. Howard, R. Sankar, F. C. Chou, C. Chamon, and M. El-Batanouny, Phys. Rev. Lett. 108, 185501 (2012). 